\title{
MIXED PROJECTION INEQUALITIES
}

\author{
BY
}

ERWIN LUTWAK

\begin{abstract}
A number of sharp geometric inequalities for polars of mixed projection bodies (zonoids) are obtained. Among the inequalities derived is a polar projection inequality that has the projection inequality of Petty as a special case. Other special cases of this polar projection inequality are inequalities (between the volume of a convex body and that of the polar of its $i$ th projection body) that are strengthened forms of the classical inequalities between the volume of a convex body and its projection measures (Quermassintegrale). The relation between the Busemann-Petty centroid inequality and the Petty projection inequality is shown to be similar to the relation that exists between the Blaschke-Santaló inequality and the affine isoperimetric inequality of affine differential geometry. Some mixed integral inequalities are derived similar in spirit to inequalities obtained by Chakerian and others.
\end{abstract}

0. Introduction. Projection bodies or zonoids (we will use the terms interchangeably) in $\mathbf{R}^{n}$ have received considerable attention in recent years (see, for example, $[6,16,25,34,36,45-49])$. They arise naturally in a number of disciplines such as functional analysis, convexity, the geometry of polytopes and stochastic geometry. Zonoids can be defined in several equivalent ways $[\mathbf{4}, \mathbf{4 2}]$, for example, as the range of a nonatomic vector $\left(\mathbf{R}^{n}\right.$-valued) measure or as the limit (with respect to the Hausdorff metric) of finite sums of segments. The polars of zonoids can be viewed as the finite-dimensional central sections of the unit ball of $L^{1}$. The work of Bolker $[4]$ and Schneider and Weil $[\mathbf{4 2}]$ provide excellent introductions to the subject of zonoids or projection bodies. (We follow the standard usage in convexity of the term projection body [42]. Thus what we refer to as a projection body is the polar of the object Bolker [4] calls a projection body.)

The purpose of this article is to present some sharp inequalities involving mixed projection bodies. Mixed projection bodies are related to projection bodies in the same way that mixed volumes are related to ordinary volumes. The mixed projection bodies are by definition more general than projection bodies. However, the class of mixed projection bodies is the same as the class of projection bodies. The definition and elementary properties of mixed projection bodies can be found in the classic volume of Bonnesen and Fenchel [5]. The support functions of mixed projection bodies were studied by Chakerian [10] (see also [43]). Schneider [36] obtained an interesting characterization of the sphere involving mixed projection bodies.

One of our objectives is to obtain a general form of the projection inequality of Petty [28] involving the volume of a convex body and that of the polar of its associ-

Received by the editors July 18, 1983.

1980 Mathematics Subject Classification. Primary 52A40; Secondary 53A15, 46B20.

Key words and phrases. Centroid body, convex body, mixed area measure, mixed volume, projection body, projection measure (Quermassintegral), zonoid. 
ated projection body. There are a number of open questions involving inequalities between the volume of a convex body and that of its projection body (see, for example, $[\mathbf{2 7}$, p. $238 ; \mathbf{2 8}$, p. $136 ; \mathbf{3 9}$, p, 385]). The general form of the Petty projection inequality that we will derive turns out to yield, among other things, strengthened forms of the classical inequalities between the volume of a convex body and its projection measures (Quermassintegrale).

We are especially interested in exploring the relation that exists between the Petty projection inequality and the Busemann-Petty centroid inequality [26]. This relation turns out to be quite surprising. It seems that the relation is very similar to the relation that is shown in $[\mathbf{2 3}]$ to exist between the Blaschke-Santaló inequality and the affine isoperimetric inequality of affine differential geometry.

Since interest in projection bodies is not confined to one discipline, we have attempted to make this article reasonably self-contained. Toward this end, we have restated some basic definitions and facts. In $\S \S 1$ and 2 we collect, for quick reference, the facts we require later on. Nothing in $\S \S 1$ and 2 is original, probably not even the presentation. References for the material presented in $\S 1$ are Bonnessen and Fenchel [5], Burago and Zalgaller [7] and Busemann [9]. References for the mixed area measures (considered in $\S 2$ ) are the original papers of Aleksandrov [1] and Fenchel and Jessen [15]. Introductions to mixed area measures can also be found in Schneider [37], Burago and Zalgaller [7], Busemann [9] and Leichtweiss [19]. Chakerian [10] is a good reference for the mixed brightness functions considered in $\S 2$. In $\S 3$, some inequalities are derived for polars of mixed projection bodies, analogous to the Aleksandrov-Fenchel and Minkowski inequalities. $\S 4$ deals with the centroid inequality of Busemann and Petty and some of its extensions. A good reference here is the article of Petty [26]. In $\S 5$, an inequality is obtained (which we call the polar centroid inequality) which is closely related to (but independent of) the Petty projection inequality and the Busemann-Petty centroid inequality. A general form of the Petty projection inequality is derived in $\S 6$. Here the relationship between the Busemann-Petty centroid inequality and the Petty projection inequality is examined. In $\S 7$, some mixed projection integrals are introduced which are closely related to integrals considered by Chakerian $[\mathbf{1 1}, \mathbf{1 2}$, 13], Chakerian and Sangwine-Yager $[14]$ and Lutwak $[\mathbf{2 1}, 22,24]$ (see also Burago and Zalgaller $[\mathbf{7}])$. By using the generalization of the Petty projection inequality obtained in $\S 6$, a geometric inequality is derived between the volumes of convex bodies and their associated mixed projection integral. A geometric inequality is obtained in $\S 8$ between the volume of a convex body and the harmonic mean of the lateral surface areas of its circumscribed right cylinders, similar to inequalities obtained by Chakerian $[\mathbf{1 1}]$ and Knothe $[\mathbf{1 8}]$.

1. Basic definitions and mixed volumes. The setting for this article will be $n$-dimensional Euclidean space $\mathbf{R}^{n}(n>2)$. We use the term convex figure when referring to a compact convex subset of $\mathbf{R}^{n}$. The term convex body is reserved for convex figures with nonempty interiors. We will use $K$ or $\bar{K}$ (possibly with subscripts) to denote a convex figure, exclusively. The space of convex figures in $\mathbf{R}^{n}$ endowed with the topology induced by the Hausdorff metric is denoted by $K^{n}$. We reserve the letter $u$ (possibly with distinguishing marks) for unit vectors, the letters $i, j, r$ for nonnegative integers, and $\lambda$ for nonnegative real numbers. 
For a convex figure $K$, we use $V(K)$ for the $n$-dimensional volume of $K$. The letter $B$ is reserved for the unit ball centered at the origin. The surface of $B$ is $S^{n-1}$, and the volume of $B$ will be denoted by $\omega_{n}$.

Associated with a convex figure $K$ is its support function $h(K, \cdot)$ defined on $\mathbf{R}^{n}$ by

$$
h(K, x)=\operatorname{Max}\{x \cdot y: y \in K\},
$$

where $x \cdot y$ is the usual inner product of $x$ and $y$ in $\mathbf{R}^{n}$. The support function $h$ of a convex figure that contains the origin is a nonnegative real-valued function on $\mathbf{R}^{n}$ with the properties

$$
h(\lambda x)=\lambda h(x) \quad \text { for } \lambda \geq 0, \quad h(x+y) \leq h(x)+h(y) .
$$

Conversely, a nonnegative real-valued function on $\mathbf{R}^{n}$ satisfying properties (1.1) is the support function of a unique convex figure (that contains the origin). We will usually be concerned with the restriction of the support function to $S^{n-1}$. We will often define a convex figure by specifying its support function $h$ on $S^{n-1}$. The homogeneous extension of $h$ to $\mathbf{R}^{n}$ by setting $h(0)=0$ and $h(x)=|x| h(x /|x|)$, for $x \neq 0$, where $|\cdot|$ denotes length, is implied. For a convex figure $K$ and a nonnegative scalar $\lambda, \lambda K$ is used to denote $\{\lambda x: x \in K\}$. If $K_{1}, \ldots, K_{r}$ are convex bodies, then the vector sum of $K_{1}, \ldots, K_{r}$ is defined by

$$
K_{1}+\cdots+K_{r}=\left\{x_{1}+\cdots+x_{r}: x_{1} \in K_{1}, \ldots, x_{r} \in K_{r}\right\} .
$$

Of basic importance is the way in which the support function behaves with respect to scalar multiplication and vector addition of convex bodies:

$$
h(\lambda K, \cdot)=\lambda h(K, \cdot), \quad h(K+\bar{K}, \cdot)=h(K, \cdot)+h(\bar{K}, \cdot) .
$$

If $K$ is a convex body that contains the origin in its interior, then we associate with $K$ its radial function $\rho(K, \cdot)$ defined on $S^{n-1}$ by

$$
\rho(K, u)=\operatorname{Max}\{\lambda>0: \lambda u \in K\} .
$$

We recall that the polar coordinate formula for volume in $\mathbf{R}^{n}$ is

$$
V(K)=\frac{1}{n} \int_{S^{n-1}} \rho(K, u)^{n} d S(u)
$$

where $d S(u)$ denotes the area element of $S^{n-1}$ at $u$.

If $K$ is a convex body that contains the origin in its interior, then the polar body of $K$ with respect to the (unit sphere centered at the) origin, $K^{*}$, can be defined by

$$
h\left(K^{*}, u\right)=\rho(K, u)^{-1} .
$$

The verification that the homogeneous extension of $h$ has the properties (1.1) is not difficult.

If $K_{1}, \ldots, K_{r}$ are convex figures and $\lambda_{1}, \ldots, \lambda_{r}$ are nonnegative real numbers, then of fundamental importance is the fact that the volume of $\lambda_{1} K_{1}+\cdots+\lambda_{r} K_{r}$ is a homogeneous polynomial in $\lambda_{1}, \ldots, \lambda_{r}$ given by

$$
V\left(\lambda_{1} K_{1}+\cdots+\lambda_{r} K_{r}\right)=\sum \lambda_{i_{1}} \cdots \lambda_{i_{n}} V\left(K_{i_{1}}, \ldots, K_{i_{n}}\right)
$$

where the sum is taken over all $n$-tuples of positive integers $\left(i_{1}, \ldots, i_{n}\right)$ whose entries do not exceed $r$. The coefficient $V\left(K_{i_{1}}, \ldots, K_{i_{n}}\right)$ is called the mixed volume 
of $K_{i_{1}}, \ldots, K_{i_{n}}$ and is uniquely determined by the requirement that it be symmetric in its arguments. The following elementary properties of mixed volumes will be used later:

$$
V\left(K_{1}, \ldots, K_{n-1}, K_{n}+\bar{K}_{n}\right)=V\left(K_{1}, \ldots, K_{n-1}, K_{n}\right)+V\left(K_{1}, \ldots, K_{n-1}, \bar{K}_{n}\right),
$$

$$
\begin{gathered}
V\left(\lambda_{1} K_{1}, \ldots, \lambda_{n} K_{n}\right)=\lambda_{1} \cdots \lambda_{n} V\left(K_{1}, \ldots, K_{n}\right), \\
V\left(x_{1}+K_{1}, \ldots, x_{n}+K_{n}\right)=V\left(K_{1}, \ldots, K_{n}\right) .
\end{gathered}
$$

If $K_{1}=\cdots=K_{n-i}=K$ and $K_{n-i+1}=\cdots=K_{n}=\bar{K}$, then the mixed volume $V\left(K_{1}, \ldots, K_{n}\right)$ is written as $V_{i}(K, \bar{K})$. If $\bar{K}=B$, then $V_{i}(K, B)$ is the $i$ th projection measure (Quermassintegral) of $K$ and is written as $W_{i}(K)$. With this notation, $W_{0}(K)=V(K)$, while $n W_{1}(K)$ is the surface area of $K, S(K)$.

If $K$ is a convex figure and $u \in S^{n-1}$, we will use $K^{u}$ to denote the image of $K$ under an orthogonal projection onto the hyperplane $E_{u}$ which passes through the origin and is orthogonal to $u$. If $K_{1}, \ldots, K_{n-1}$ are convex figures and $u \in$ $S^{n-1}$, then the mixed volume of the convex figures $K_{1}^{u}, \ldots, K_{n-1}^{u}$ in the $(n-1)$ dimensional space $E_{u}$ will be denoted by $v\left(K_{1}^{u}, \ldots, K_{n-1}^{u}\right)$. The relation between the mixed volumes is

$$
v\left(K_{1}^{u}, \ldots, K_{n-1}^{u}\right)=n V\left(K_{1}, \ldots, K_{n-1},\langle u\rangle\right),
$$

where $\langle u\rangle$ denotes the closed line segment $\{\lambda u:|\lambda| \leq 1 / 2\}$. If $K_{1}=\cdots=K_{n-1-i}=$ $K$ and $K_{n-i}=\cdots=K_{n-1}=\bar{K}$, then $v\left(K_{1}^{u}, \ldots, K_{n-1}^{u}\right)$ is written $v_{i}\left(K^{u}, \bar{K}^{u}\right)$. If $\bar{K}=B$, then $v_{i}\left(K^{u}, B^{u}\right)$ is the $i$ th projection measure of $K^{u}$ in $E_{u}$ and is written $w_{i}\left(K^{u}\right)$.

The inequalities of Minkowski and of Aleksandrov and Fenchel are of fundamental importance in the theory of mixed volumes. The Minkowski inequality for convex bodies $K, \bar{K}$ in $\mathbf{R}^{n}$ is

$$
V(K)^{n-1} V(\bar{K}) \leq V_{1}^{n}(K, \bar{K}),
$$

with equality if and only if $K$ and $\bar{K}$ are homothetic. The Aleksandrov-Fenchel inequality for convex figures $K_{1}, \ldots, K_{n}$ in $\mathbf{R}^{n}$ is

$$
V\left(K_{1}, K_{1}, K_{3}, \ldots, K_{n}\right) V\left(K_{2}, K_{2}, K_{3}, \ldots, K_{n}\right) \leq V^{2}\left(K_{1}, K_{2}, K_{3}, \ldots, K_{n}\right) .
$$

The equality conditions in the Aleksandrov-Fenchel inequality are, in general, unknown (see $[\mathbf{4 0}]$ ). By repeated applications of the Aleksandrov-Fenchel inequality and final applications of the Minkowski inequality, the following inequality for convex bodies $K_{1}, \ldots, K_{n}$ in $\mathbf{R}^{n}$ is obtained:

$$
V\left(K_{1}\right) \cdots V\left(K_{n}\right) \leq V^{n}\left(K_{1}, \ldots, K_{n}\right),
$$

with equality if and only if the $K_{i}$ are homothetic. If we take $K_{n}=B$ in (1.7), we obtain

$$
\omega_{n} V\left(K_{1}\right) \cdots V\left(K_{n-1}\right) \leq V^{n}\left(K_{1}, \ldots, K_{n-1}, B\right),
$$

with equality if and only if the $K_{i}$ are balls. If, for $0 \leq i<n-1$, we take $K_{1}=\cdots=K_{n-i-1}=K$ and $K_{n-i}=\cdots=K_{n-1}=B$ in (1.8), we obtain

$$
\omega_{n}^{i+1} V(K)^{n-i-1} \leq W_{i+1}^{n}(K),
$$


with equality if and only if $K$ is a ball. This is the classical inequality between the volume of a convex body and its projection measures. The case $i=0$ is the isepiphanic inequality (between the volume and surface area of a convex body), while the case $i=n-2$ is the Urysohn inequality (between the volume and mean width of a convex body).

2. Mixed brightness. The construction of the mixed area measures can be found in the classic works of Aleksandrov [1] and Fenchel and Jessen [15]. From (1.5), one sees that for fixed convex bodies $K_{1}, \ldots, K_{n-1}$, one can view $V\left(K_{1}, \ldots, K_{n-1}, \cdot\right)$ as a functional on $\mathcal{K}^{n}$. If each $K \in \mathcal{K}^{n}$ is identified with its support function on $S^{n-1}$, then $V\left(K_{1}, \ldots, K_{n-1}, \cdot\right)$ when extended (in a unique way) is a continuous functional on the vector space of continuous functions on $S^{n-1}$, endowed with the max norm (viz. (1.2)). The Riesz representation theorem can then be used to yield a unique positive Borel measure on $S^{n-1}$, denoted by $S\left(K_{1}, \ldots, K_{n-1} ; \cdot\right)$ and called the mixed area measure of $K_{1}, \ldots, K_{n-1}$, such that for each $K_{n} \in K^{n}$

$$
V\left(K_{1}, \ldots, K_{n-1}, K_{n}\right)=\frac{1}{n} \int_{S^{n-1}} h\left(K_{n}, u\right) d S\left(K_{1}, \ldots, K_{n-1} ; u\right),
$$

where the integration is with respect to the measure $S\left(K_{1}, \ldots, K_{n-1} ; \cdot\right)$ on $S^{n-1}$.

From the corresponding property of the mixed volumes, it follows that the mixed area function $S\left(K_{1}, \ldots, K_{n-1} ; \cdot\right)$ is symmetric in its (first $\left.n-1\right)$ arguments. Also from (1.5), it follows that

$$
\begin{aligned}
& S\left(\lambda_{1} K_{1}, \ldots, \lambda_{n-1} K_{n-1} ; \cdot\right)=\lambda_{1} \cdots \lambda_{n-1} S\left(K_{1}, \ldots, K_{n-1} ; \cdot\right), \\
& S\left(x_{1}+K_{1}, \ldots, x_{n-1}+K_{n-1} ; \cdot\right)=S\left(K_{1}, \ldots, K_{n-1} ; \cdot\right) .
\end{aligned}
$$

For convex bodies $K_{1}, \ldots, K_{n-1}$ and a direction $u \in S^{n-1}$, the mixed brightness of $K_{1}, \ldots, K_{n-1}$ in the direction $u, \sigma\left(K_{1}, \ldots, K_{n-1} ; u\right)$, can be defined by

$$
\sigma\left(K_{1}, \ldots, K_{n-1} ; u\right)=n V\left(K_{1}, \ldots, K_{n-1},\langle u\rangle\right) .
$$

Since $h(\langle u\rangle, \bar{u})=\frac{1}{2}|u \cdot \bar{u}|$, from $(2.1)$ one obtains

$$
\sigma\left(K_{1}, \ldots, K_{n-1} ; u\right)=\frac{1}{2} \int_{S^{n-1}}|u \cdot \bar{u}| d S\left(K_{1}, \ldots, K_{n-1} ; \bar{u}\right) .
$$

By using (1.6), the mixed brightness of $K_{1}, \ldots, K_{n-1}$ in the direction $u$ can be written as

$$
\sigma\left(K_{1}, \ldots, K_{n-1} ; u\right)=v\left(K_{1}^{u}, \ldots, K_{n-1}^{u}\right) .
$$

If $\sigma\left(K_{1}, \ldots, K_{n-1} ; u\right)$ is constant for all (independent of) $u \in S^{n-1}$, we will say the bodies $K_{1}, \ldots, K_{n-1}$ have constant joint brightness.

If $K_{1}=\cdots=K_{n-i-1}=K$ and $K_{n-i}=\cdots=K_{n-1}=\bar{K}$, then the mixed brightness $\sigma\left(K_{1}, \ldots, K_{n-1} ; u\right)$ is written as $\sigma_{i}(K, \bar{K} ; u)$. If $\bar{K}=B$, then instead of $\sigma_{i}(K, B ; u)$, we write $\sigma_{i}(K, u)$, and $\sigma_{i}(K, u)$ is called the $(n-i-1)$-girth of $K$ in the direction $u$. If $i=0$, then we write $\sigma(K, u)$ rather than $\sigma_{0}(K, u)$, and $\sigma(K, u)$ is simply called the brightness of $K$ in the direction $u$. From (2.5) we have

$$
\sigma_{i}(K, \bar{K} ; u)=v_{i}\left(K^{u}, \bar{K}^{u}\right), \quad \sigma_{i}(K, u)=w_{i}\left(K^{u}\right), \quad \sigma(K, u)=v\left(K^{u}\right) .
$$


Since $h(B, u)=1$, from $(2.1)$ it follows that

$$
V\left(K_{1}, \ldots K_{n-1}, B\right)=\frac{1}{n} \int_{S^{n-1}} d S\left(K_{1}, \ldots, K_{n-1} ; u\right) .
$$

By combining (2.4) and (2.5) integrating both sides over $S^{n-1}$, interchanging the order of integration and using (2.7), one obtains

$$
\omega_{n-1} V\left(K_{1}, \ldots, K_{n-1}, B\right)=\frac{1}{n} \int_{S^{n-1}} v\left(K_{1}^{u}, \ldots, K_{n-1}^{u}\right) d S(u) .
$$

For $K_{1}=\cdots=K_{n-1-i}=K$ and $K_{n-i}=\cdots=K_{n-1}=B$, (2.8) becomes

$$
\omega_{n-1} W_{i+1}(K)=\frac{1}{n} \int_{S^{n-1}} w_{i}\left(K^{u}\right) d S(u) .
$$

3. Mixed projection bodies. If $K_{1}, \ldots, K_{n-1}$ are convex bodies, then the mixed projection body of $K_{1}, \ldots, K_{n-1}$ is denoted by $\Pi\left(K_{1}, \ldots, K_{n-1}\right)$, and is defined by

$$
h\left(\Pi\left(K_{1}, \ldots, K_{n-1}\right), u\right)=\sigma\left(K_{1}, \ldots, K_{n-1} ; u\right) .
$$

From (2.4) and (3.1), it is easy to see that $\Pi\left(K_{1}, \ldots, K_{n-1}\right)$ must be a convex body that is symmetric with respect to the origin. If $K_{1}=\cdots=K_{n-1}=B$, then a simple calculation shows

$$
\Pi(B, \ldots, B)=\omega_{n-1} B .
$$

From the corresponding properties of the mixed area measures (2.2) and (2.4) or from the corresponding properties of the $(n-1)$-dimensional mixed volumes and (2.3) or $(2.5)$, it follows that $\Pi\left(K_{1}, \ldots, K_{n-1}\right)$ is symmetric in its arguments, and that we have

$$
\begin{aligned}
& \Pi\left(\lambda_{1} K_{1}, \ldots, \lambda_{n-1} K_{n-1}\right)=\lambda_{1} \cdots \lambda_{n-1} \Pi\left(K_{1}, \ldots, K_{n-1}\right), \\
& \Pi\left(x_{1}+K_{1}, \ldots, x_{n-1}+K_{n-1}\right)=\Pi\left(K_{1}, \ldots, K_{n-1}\right) .
\end{aligned}
$$

We use $\Pi^{*}\left(K_{1}, \ldots, K_{n-1}\right)$ to denote the polar body of $\Pi\left(K_{1}, \ldots, K_{n-1}\right)$.

If, for $0 \leq i<n, K_{1}=\cdots=K_{n-1-i}=K$, while $K_{n-i}=\cdots=K_{n-1}=\bar{K}$, then $\Pi\left(K_{1}, \ldots, K_{n-1}\right)$ will be written as $\Pi_{i}(K, \bar{K})$. If $\bar{K}=B$, then $\Pi_{i}(K, B)$ is called the $i$ th projection body of $K$ and is denoted $\Pi_{i} K$. We write $\Pi_{0} K$ as $\Pi K$. Rather than $\left(\Pi_{i} K\right)^{*}$ and $(\Pi K)^{*}$, we will simply write $\Pi_{i}^{*} K$ and $\Pi^{*} K$, respectively.

From (3.1) and (2.6), we have

$$
\begin{aligned}
& h\left(\Pi_{i}(K, \bar{K}), u\right)=\sigma_{i}(K, \bar{K} ; u)=v_{i}\left(K^{u}, \bar{K}^{u}\right), \\
& h\left(\Pi_{i} K, u\right)=\sigma_{i}(K, u)=w_{i}\left(K^{u}\right), \\
& h(\Pi K, u)=\sigma(K, u)=v\left(K^{u}\right) .
\end{aligned}
$$

If we use (1.4), (3.3) and the polar coordinate formula for volume (1.3), we obtain

$$
\begin{aligned}
& V\left(\Pi_{i}^{*}(K, \bar{K})\right)=\frac{1}{n} \int_{S^{n-1}} v_{i}\left(K^{u}, \bar{K}^{u}\right)^{-n} d S(u), \\
& V\left(\Pi_{i}^{*} K\right)=\frac{1}{n} \int_{S^{n-1}} w_{i}\left(K^{u}\right)^{-n} d S(u), \\
& V\left(\Pi^{*} K\right)=\frac{1}{n} \int_{S^{n-1}} v\left(K^{u}\right)^{-n} d S(u) .
\end{aligned}
$$


If $K, \bar{K}$ are convex bodies in $\mathbf{R}^{n}$ and $u \in S^{n-1}$, then from the Minkowski (mixed volume) inequality, we have

$$
v\left(K^{u}\right)^{n-2} v\left(\bar{K}^{u}\right) \leq v_{1}\left(K^{u}, \bar{K}^{u}\right)^{n-1},
$$

with equality if and only if $K^{u}$ and $\bar{K}^{u}$ are homothetic.

For positive real numbers $p, q$ such that $p^{-1}+q^{-1}=1$ and for positive continuous functions $f, g$ on $S^{n-1}$, the Hölder integral inequality [17, p. 140] states that

$$
\int_{S^{n-1}} f(u) g(u) d S(u) \leq\left[\int_{S^{n-1}} f^{p}(u) d S(u)\right]^{1 / p}\left[\int_{S^{n-1}} g^{q}(u) d S(u)\right]^{1 / q},
$$

with equality if and only if $f^{p}$ and $g^{q}$ are proportional. (Two positive functions $F, G$ are called proportional if there exists a constant $c$ such that $F(u)=c G(u)$ for all $u$.)

If we take $p=(n-1) /(n-2), q=n-1, f(u)=v\left(K^{u}\right)^{-n(n-2) /(n-1)}$ and $g(u)=v\left(\bar{K}^{u}\right)^{-n /(n-1)}$, then the Hölder inequality in conjunction with (3.5) and (3.4) will yield

(3.6) THEOREM. If $K, \bar{K}$ are convex bodies in $\mathbf{R}^{n}$, then

$$
V^{n-1}\left(\Pi_{1}^{*}(K, \bar{K})\right) \leq V^{n-2}\left(\Pi^{*} K\right) V\left(\Pi^{*} \bar{K}\right),
$$

with equality if and only if $K$ and $\bar{K}$ are homothetic.

The conditions for equality follow from the conditions for equality in (3.5) and a theorem of Süss [44] (see also Aleksandrov [2] or Rogers [31]) which states that $K$ and $\bar{K}$ are homothetic if and only if, for all $u \in S^{n-1}, K^{u}$ and $\bar{K}^{u}$ are homothetic.

If $K_{1}, \ldots, K_{n-1}$ are convex bodies in $\mathbf{R}^{n}$ and $u \in S^{n-1}$, then from (1.4), (2.5), (3.1) and the Aleksandrov-Fenchel inequality we have

$$
\begin{aligned}
& \rho\left(\Pi^{*}\left(K_{1}, K_{2}, K_{3}, \ldots, K_{n-1}\right), u\right)^{n} \\
& \quad \leq \rho\left(\Pi^{*}\left(K_{1}, K_{1}, K_{3}, \ldots, K_{n-1}\right), u\right)^{n / 2} \rho\left(\Pi^{*}\left(K_{2}, K_{2}, K_{3}, \ldots, K_{n-1}\right), u\right)^{n / 2} .
\end{aligned}
$$

If we integrate both sides of this inequality, apply the Schwarz inequality $[\mathbf{1 7}, \mathrm{p}$. 132] to the two functions on the right, and use the polar coordinate formula for volume (1.3), we obtain

(3.7) THEOREM. If $K_{1}, \ldots, K_{n-1}$ are convex bodies in $\mathbf{R}^{n}$, then

$$
\begin{aligned}
V^{2}\left(\Pi^{*}\left(K_{1}, K_{2}, K_{3}, \ldots, K_{n-1}\right)\right) \\
\quad \leq V\left(\Pi^{*}\left(K_{1}, K_{1}, K_{3}, \ldots, K_{n-1}\right)\right) V\left(\Pi^{*}\left(K_{2}, K_{2}, K_{3}, \ldots, K_{n-1}\right)\right) .
\end{aligned}
$$

By repeated applications of Theorem (3.7) with final applications of Theorem (3.6), we obtain

(3.8) THEOREM. If $K_{1}, \ldots, K_{n-1}$ are convex bodies in $\mathbf{R}^{n}$, then

$$
V^{n-1}\left(\Pi^{*}\left(K_{1}, \ldots, K_{n-1}\right)\right) \leq V\left(\Pi^{*} K_{1}\right) \cdots V\left(\Pi^{*} K_{n-1}\right),
$$

with equality if and only if the $K_{i}$ are homothetic.

If for $0<i<n-1$, we take $K_{1}=\cdots=K_{n-i-1}=K$ and $K_{n-i}=\cdots=K_{n-1}=$ $B$ in Theorem (3.8), we obtain the following inequality between the volume of the polar of the projection body of $K$ and the volume of the polar of its $i$ th projection body: 
(3.9) Corollary. If $K$ is a convex body in $\mathbf{R}^{n}$, and $0<i<n-1$, then

$$
\omega_{n-1}^{i n} V^{n-1}\left(\Pi_{i}^{*} K\right) \leq \omega_{n}^{i} V\left(\Pi^{*} K\right)^{n-i-1},
$$

with equality if and only if $K$ is a ball.

From (2.3) and (3.1) it follows that for convex bodies $K_{1}, \ldots, K_{n-1}$, and $\bar{K}_{1}, \ldots$, $\bar{K}_{n-1}$ we have $\Pi\left(K_{1}, \ldots, K_{n-1}\right)=\Pi\left(\bar{K}_{1}, \ldots, \bar{K}_{n-1}\right)$ if and only if

$$
V\left(K_{1}, \ldots, K_{n-1},\langle u\rangle\right)=V\left(\bar{K}_{1}, \ldots, \bar{K}_{n-1},\langle u\rangle\right)
$$

for all $u \in S^{n-1}$. However, $\Pi\left(K_{1}, \ldots, K_{n-1}\right)=\Pi\left(\bar{K}_{1}, \ldots, \bar{K}_{n-1}\right)$ implies a good deal more. The following is a trivial generalization of Theorem 1 of $[\mathbf{2 7}]$.

(3.10) THEOREM. If $K_{1}, \ldots, K_{n-1}, \bar{K}_{1}, \ldots, \bar{K}_{n-1}$ are convex bodies in $\mathbf{R}^{n}$ such that $\Pi\left(K_{1}, \ldots, K_{n-1}\right)=\Pi\left(\bar{K}_{1}, \ldots, \bar{K}_{n-1}\right)$, then

$$
V\left(K_{1}, \ldots, K_{n-1}, K\right)=V\left(\bar{K}_{1}, \ldots, \bar{K}_{n-1}, K\right)
$$

for any convex body $K$ in $\mathbf{R}^{n}$ that is centrally symmetric.

PROOF. This is a standard argument (see, for example, $[\mathbf{1 0}$, p. 28, or 27, p. $234]$ ). If $K$ is centrally symmetric and sufficiently smooth, then there exists (see [5. p. 29]) a continuous function $g$ on $S^{n-1}$ such that the support function of $K$ has the representation

$$
h(K, u)=\int_{S^{n-1}}|u \cdot \bar{u}| g(\bar{u}) d S(\bar{u})
$$

If we combine this with (2.1), change the order of integration and use (2.4) and (3.1) we obtain $V\left(K_{1}, \ldots, K_{n-1}, K\right)=V\left(\bar{K}_{1}, \ldots, \bar{K}_{n-1}, K\right)$. A standard approximation argument (that makes use of the continuity of the mixed volumes) can now be used to obtain the same result for an arbitrary (convex body) $K$ that is centrally symmetric.

As an aside we note that it is not difficult to show that if $K_{1}, \ldots, K_{r}$ are convex bodies in $\mathbf{R}^{n}$ and $\lambda_{1}, \ldots, \lambda_{r}$ are nonnegative real numbers, then for the projection body of $\lambda_{1} K_{1}+\cdots+\lambda_{r} K_{r}$ one has

$$
\Pi\left(\lambda_{1} K_{1}+\cdots+\lambda_{r} K_{r}\right)=\sum \lambda_{i_{1}} \cdots \lambda_{i_{n-1}} \Pi\left(K_{i_{1}}, \ldots, K_{i_{n-1}}\right),
$$

where the sum on the right is the Minkowski sum taken over all $(n-1)$-tuples of positive integers $\left(i_{1}, \ldots, i_{n-1}\right)$ whose entries do not exceed $r$.

4. Centroid inequalities. If $K$ is a convex body with the origin in its interior, then associated with $K$ is its centroid body $[\mathbf{2 6}]$, with respect to the origin, $\Gamma K$, defined by

$$
h(\Gamma K, u)=\frac{1}{V(K)} \int_{K}|u \cdot x| d x,
$$

where the integration is over the interior of $K$ and $d x$ is the volume element at $x \in K$. For our purposes, the following equivalent definition of $\Gamma K$ (see [26]) will be more useful:

$$
h(\Gamma K, u)=\frac{1}{(n+1) V(K)} \int_{S^{n-1}}|u \cdot \bar{u}| \rho(K, \bar{u})^{n+1} d S(\bar{u}) .
$$


It is easy to see that (the homogeneous extension of) $h(\Gamma K, \cdot)$ has properties $(1.1)$ and hence defines a convex body. It follows immediately from definition (4.1) that $\Gamma K$ is symmetric with respect to the origin. A simple calculation shows that

$$
\Gamma B=\left(2 \omega_{n-1} /(n+1) \omega_{n}\right) B .
$$

Let $T\left(x_{1}, \ldots, x_{n}\right)$ denote the $n$-dimensional volume of the simplex in $\mathbf{R}^{n}$ whose vertices consist of the origin and the points $x_{1}, \ldots, x_{n}$. Busemann $[8, p .11]$ has established the inequality

$$
2 \omega_{n+1}^{n-1} V(K)^{n+1} \leq \omega_{n}^{n+1}(n+1) ! \int_{K} \cdots \int_{K} T\left(x_{1}, \ldots, x_{n}\right) d x_{1} \cdots d x_{n},
$$

with equality if and only if $K$ is an ellipsoid centered at the origin. Petty [26] has shown that if $K$ is a convex body that contains the origin in its interior, then this inequality of Busemann can be viewed as an inequality between the volume of $K$ and the volume of the centroid body of $K$. Specifically, Petty has proved that if $K$ is a convex body that contains the origin in its interior, then

$$
V(K) \leq\left((n+1) \omega_{n} / 2 \omega_{n-1}\right)^{n} V(\Gamma K),
$$

with equality if and only if $K$ is an ellipsoid centered at the origin. We will refer to this inequality as the Busemann-Petty centroid inequality.

We note that the convexity of $K$ is not an essential feature of the BusemannPetty centroid inequality (see $[\mathbf{2 6}]$ ); however, we shall restrict our attention to inequalities between convex bodies.

If we combine inequality (1.7) with the Busemann-Petty centroid inequality, we obtain an inequality which we will refer to as the general centroid inequality:

(4.3) THEOREM. If $K_{1}, \ldots, K_{n}$ are convex bodies in $\mathbf{R}^{n}$ and $K_{n}$ contains the origin in its interior, then

$$
V\left(K_{1}\right) \cdots V\left(K_{n-1}\right) V\left(K_{n}\right) \leq\left((n+1) \omega_{n} / 2 \omega_{n-1}\right)^{n} V^{n}\left(K_{1}, \ldots, K_{n-1}, \Gamma K_{n}\right),
$$

with equality if and only if $K_{n}$ is an ellipsoid centered at the origin and each $K_{i}$ is homothetic to $K_{n}$.

For the equality conditions, we need merely observe that if $E$ is an ellipsoid centered at the origin, then $\Gamma E$ is homothetic to (in fact, a dilation of) $E$.

A special case of this inequality which is closely related (as will be seen later) to the projection inequality of Petty is given in

(4.4) COROLlaRY. If $K$ and $\bar{K}$ are convex bodies in $\mathbf{R}^{n}$ and $\bar{K}$ contains the origin in its interior, then

$$
V(K)^{n-1} V(\bar{K}) \leq\left((n+1) \omega_{n} / 2 \omega_{n-1}\right)^{n} V_{1}^{n}(K, \Gamma \bar{K}),
$$

with equality if and only if $\bar{K}$ is an ellipsoid centered at the origin and $K$ is homothetic to $\bar{K}$.

We observe that the special case $K=\Gamma \bar{K}$ in Corollary (4.4) is the BusemannPetty centroid inequality. 
5. Polar centroid inequalities. We now derive a polar inequality which is related to but will be derived independently of the general centroid inequality (Theorem (4.3)).

Let $K_{1}, \ldots, K_{n}$ be arbitrary convex bodies in $\mathbf{R}^{n}$, such that $K_{n}$ contains the origin in its interior. From (2.1), we have

$$
V\left(K_{1}, \ldots, K_{n-1}, \Gamma K_{n}\right)=\frac{1}{n} \int_{S^{n-1}} h\left(\Gamma K_{n}, \bar{u}\right) d S\left(K_{1}, \ldots, K_{n-1} ; \bar{u}\right) .
$$

If we combine this with (4.1), we obtain

$$
\begin{aligned}
& (n+1) V\left(K_{n}\right) V\left(K_{1}, \ldots, K_{n-1}, \Gamma K_{n}\right) \\
& \quad=\frac{1}{n} \int_{S^{n-1}} d S\left(K_{1}, \ldots, K_{n-1} ; \bar{u}\right) \int_{S^{n-1}}|u \cdot \bar{u}| \rho\left(K_{n}, u\right)^{n+1} d S(u) .
\end{aligned}
$$

We interchange the order of integration, use (2.4) and obtain

$$
\begin{aligned}
(n+1) V\left(K_{h}\right) V\left(K_{1}, \ldots, K_{n-1}, \Gamma K_{n}\right) & \\
= & \frac{2}{n} \int_{S^{n-1}} \sigma\left(K_{1}, \ldots, K_{n-1} ; u\right) \rho\left(K_{n}, u\right)^{n+1} d S(u) .
\end{aligned}
$$

If in the Hölder inequality we take $p=(n+1) / n, q=n+1$,

$$
f(u)=\rho\left(K_{n}, u\right)^{n} \sigma\left(K_{1}, \ldots, K_{n-1} ; u\right)^{n /(n+1)},
$$

and

$$
g(u)=\sigma\left(K_{1}, \ldots, K_{n-1} ; u\right)^{-n /(n+1)},
$$

we obtain

$$
\begin{aligned}
{\left[\int_{S^{n-1}} \rho\left(K_{n}, u\right)^{n} d S(u)\right]^{n+1} \leq } & {\left[\int_{S^{n-1}} \rho\left(K_{n}, u\right)^{n+1} \sigma\left(K_{1}, \ldots, K_{n-1} ; u\right) d S(u)\right]^{n} } \\
& \times\left[\int_{S^{n-1}} \sigma\left(K_{1}, \ldots, K_{n-1} ; u\right)^{-n} d S(u)\right]
\end{aligned}
$$

with equality if and only if $\rho\left(K_{n}, u\right) \sigma\left(K_{1}, \ldots, K_{n-1} ; u\right)$ is constant. From the polar coordinate formula for volume (1.3), we see that the integral on the left of the inequality is simply $n V\left(K_{n}\right)$. From definitions (3.1) and (1.4) and the polar coordinate formula for volume, we see that the second integral on the right of the inequality is $n V\left(\Pi^{*}\left(K_{1}, \ldots, K_{n-1}\right)\right)$. If we combine this with (5.1), we obtain

(5.2) THEOREM. If $K_{1}, \ldots, K_{n}$ are convex bodies in $\mathbf{R}^{n}$, and $K_{n}$ contains the origin in its interior, then

$$
V\left(K_{n}\right) \leq\left(\frac{n+1}{2}\right)^{n} V^{n}\left(K_{1}, \ldots, K_{n-1}, \Gamma K_{n}\right) V\left(\Pi^{*}\left(K_{1}, \ldots, K_{n-1}\right)\right),
$$

with equality if and only if $K_{n}$ is a dilation of $\Pi^{*}\left(K_{1}, \ldots, K_{n-1}\right)$.

To obtain the conditions for equality, we observe that

$$
\rho\left(K_{n}, u\right) \sigma\left(K_{1}, \ldots, K_{n-1} ; u\right)=h\left(\Pi\left(K_{1}, \ldots, K_{n-1}\right), u\right) / h\left(K_{n}^{*}, u\right),
$$

and this is constant if and only if $K_{n}^{*}$ is a dilation of $\Pi\left(K_{1}, \ldots, K_{n-1}\right)$, or, equivalently, if and only if $K_{n}$ is a dilation of $\Pi^{*}\left(K_{1}, \ldots, K_{n-1}\right)$. 
We will refer to the inequality of Theorem (5.2) as the polar centroid inequality. We note, again, that while the polar centroid inequality is related to the general centroid inequality, it is easily obtained without reference to the general centroid inequality or the Busemann-Petty centroid inequality.

Of interest is the special case $K_{n}=B$ of the polar centroid inequality:

(5.3) COROLlaRY. If $K_{1}, \ldots, K_{n-1}$ are convex bodies in $\mathbf{R}^{n}$, then

$$
\omega_{n}^{n+1} \leq \omega_{n-1}^{n} V^{n}\left(K_{1}, \ldots, K_{n-1}, B\right) V\left(\Pi^{*}\left(K_{1}, \ldots, K_{n-1}\right)\right),
$$

with equality if and only if $K_{1}, \ldots, K_{n-1}$ have constant joint brightness.

If we combine Theorem (3.8) with Corollary (5.3), we obtain

(5.4) COROLlaRY. If $K_{1}, \ldots, K_{n-1}$ are convex bodies in $\mathbf{R}^{n}$, then

$$
\omega_{n}^{n+1} \leq \omega_{n-1}^{n} V^{n}\left(K_{1}, \ldots, K_{n-1}, B\right) V\left(\Pi^{*} K_{1}\right)^{1 /(n-1)} \ldots V\left(\Pi^{*} K_{n-1}\right)^{1 /(n-1)},
$$

with equality if and only if the $K_{i}$ are homothetic bodies of constant brightness.

Another special case of the polar centroid inequality, that we require later, is the case where $K_{1}=\cdots=K_{n-i-1}=K$ and $K_{n-i}=\cdots=K_{n-1}=K_{n}=B$ :

(5.5) COROLlaRY. If $K$ is a convex body in $\mathbf{R}^{n}$, and $0 \leq i<n-1$, then

$$
\omega_{n}^{n+1} \leq \omega_{n-1}^{n} W_{i+1}^{n}(K) V\left(\Pi_{i}^{*} K\right)
$$

with equality if and only if $K$ has constant $(n-i-1)$-girth.

The case $i=0$ of Corollary (5.5) can be found in Petty [28].

The case of the polar centroid inequality with $K_{1}=\cdots=K_{n-1}=K$ and $K_{n}=\bar{K}$ is another inequality related to the Petty projection inequality that will be needed later:

(5.6) COROLlARY. If $K$ and $\bar{K}$ are convex bodies in $\mathbf{R}^{n}$ and $\bar{K}$ contains the origin in its interior, then

$$
V(\bar{K}) \leq\left(\frac{n+1}{2}\right)^{n} V_{1}^{n}(K, \Gamma \bar{K}) V\left(\Pi^{*} K\right),
$$

with equality if and only if $\bar{K}$ is a dilation of $\Pi^{*} K$.

As an aside we note that $\bar{K}$ can be a dilation of $\Pi^{*} K$ only if $\bar{K}$ is symmetric with respect to the origin. For $\bar{K}$ symmetric with respect to the origin, Petty [28] has shown that the requirement that $\bar{K}$ be a dilation of $\Pi^{*} K$ is equivalent to the requirement that $K$ have constant relative brightness with respect to $\bar{K}$ (relative brightness in the sense of $[\mathbf{2 8}]$ rather than in the sense of $[\mathbf{1 0}])$.

If we take $K_{n}$ to be $\Pi^{*}\left(K_{1}, \ldots, K_{n-1}\right)$, we obtain equality in Theorem (5.2), and hence we have

(5.7) COROLlaRY. If $K_{1}, \ldots, K_{n-1}$ are convex bodies in $\mathbf{R}^{n}$, then

$$
V\left(K_{1}, \ldots, K_{n-1}, \Gamma \Pi^{*}\left(K_{1}, \ldots, K_{n-1}\right)\right)=2 /(n+1) \text {. }
$$

6. Polar projection inequalities. By using Corollary (5.7) a general form of the Petty projection inequality is now easily obtained by taking $K_{n}$ to be $\Pi^{*}\left(K_{1}, \ldots, K_{n-1}\right)$ in the general centroid inequality. 
(6.1) THEOREM. If $K_{1}, \ldots, K_{n-1}$ are convex bodies in $\mathbf{R}^{n}$, then

$$
V\left(K_{1}\right) \cdots V\left(K_{n-1}\right) V\left(\Pi^{*}\left(K_{1}, \ldots, K_{n-1}\right)\right) \leq\left(\omega_{n} / \omega_{n-1}\right)^{n},
$$

with equality if and only if the $K_{i}$ are homothetic ellipsoids.

We will refer to this inequality as the polar projection inequality. To obtain the equality conditions in the polar projection inequality from the equality conditions in the general centroid inequality, and for later use as well, we require the following trivial result.

(6.2) LEMMA. If $E_{1}, \ldots, E_{n-1}$ are homothetic ellipsoids, $\Pi^{*}\left(E_{1}, \ldots, E_{n-1}\right)$ is an ellipsoid homothetic to each of the $E_{i}$.

ProOF. Since the $E_{i}$ are homothetic ellipsoids, there exists an ellipsoid $E$, centered at the origin, such that for all $i, E_{i}=x_{i}+\lambda_{i} E$. Let $T$ be the central unimodular affinity that maps $E$ into a ball. It follows trivially, since $T E$ is a ball, that $T E$ is a dilation of $\Pi^{*} T E$. However, $\Pi^{*} T E=T \Pi^{*} E$ (see, for example, Theorem 3 in $[\mathbf{2 7}]$ ). Since $T E$ is a dilation of $T \Pi^{*} E, E$ must be a dilation of $\Pi^{*} E$.

From (3.2) and (1.4), it follows that

$$
\Pi^{*}\left(E_{1}, \ldots, E_{n-1}\right)=\left(\lambda_{1} \cdots \lambda_{n-1}\right)^{-1} \Pi^{*}(E, \ldots, E)=\left(\lambda_{1} \cdots \lambda_{n-1}\right)^{-1} \Pi^{*} E,
$$

and, hence, that $\Pi^{*}\left(E_{1}, \ldots, E_{n-1}\right)$ is homothetic to each of the $E_{i}$.

From Corollary (5.3), it is easy to see that the polar projection inequality (Theorem (6.1)) makes a stronger statement than (implies) the classical inequality (1.8).

We observe that replacing the quantity $V\left(K_{1}\right) \cdots V\left(K_{n-1}\right)$ in the polar projection inequality by the larger quantity $V^{n}\left(K_{1}, \ldots, K_{n-1}, B\right) / \omega_{n}$ reverses the sign of the inequality and results in the inequality of Corollary (5.3).

If we take $K_{1}=\cdots=K_{n-1}=K$ in the polar projection inequality, we have the Petty projection inequality:

(6.3) COROllary. If $K$ is a convex body in $\mathbf{R}^{n}$, then

$$
V(K)^{n-1} V\left(\Pi^{*} K\right) \leq\left(\omega_{n} / \omega_{n-1}\right)^{n},
$$

with equality if and only if $K$ is an ellipsoid.

This inequality was obtained by Petty [28]. As shown by Petty (and as can be seen by looking at Corollary (5.5) with $i=0$ ), this inequality makes a stronger statement than (implies) the classical isepiphanic inequality (between the volume and surface area of a convex body),

$$
\omega_{n} V(K)^{n-1} \leq W_{1}(K)^{n},
$$

with equality if and only if $K$ is a ball. The Petty projection inequality was used to obtain a number of consequent geometric inequalities in [24]. Schneider [41] has recently found a surprising application of the Petty projection inequality to an extremal problem in stochastic geometry.

We note that if one starts with the Petty projection inequality, then another way to obtain the polar projection inequality (along with the conditions for equality) is to combine Theorem (3.8) with the Petty projection inequality. The advantage of the former method is that one need not start with the Petty projection inequality, but rather, one obtains the Petty projection inequality as a special case. 
The relation between the Busemann-Petty centroid inequality and the Petty projection inequality turns out to be quite surprising. The derivation in [28] of the Petty projection inequality uses, among other things, the Busemann-Petty centroid inequality. A quick proof of the Petty projection inequality (with the conditions for equality) can be obtained by combining the Minkowski (mixed volume) inequality and the Busemann-Petty centroid inequality to obtain Corollary (4.4), and then taking $\bar{K}$ to be $\Pi^{*} K$. (We note that this choice for $\bar{K}$ is precisely the one that will yield equality in Corollary (5.6).) What is quite surprising, however, is that if we were to start with the Petty projection inequality and combine it with the polar centroid inequality of Corollary (5.6) (which depends only on the Hölder inequality), we would obtain, in turn, the general version of the Busemann-Petty centroid inequality of Corollary (4.4), along with the conditions for equality. This parallels very closely the relation that is shown in $[\mathbf{2 3}]$ to exist between the affine isoperimetric inequality of affine differential geometry [3] and the Blaschke-Santaló inequality $[30,32,33]$ (see also $[38]$ ).

If for $0<i<n-1$, we take $K_{1}=\cdots=K_{n-i-1}=K$ and $K_{n-i}=\cdots=K_{n-1}=$ $B$ in the polar projection inequality, we obtain

(6.4) CoRollaRY. If $K$ is a convex body in $\mathbf{R}^{n}$ and $0<i<n-1$, then

$$
\omega_{n-1}^{n} V(K)^{n-i-1} V\left(\Pi_{i}^{*} K\right) \leq \omega_{n}^{n-i},
$$

with equality if and only if $K$ is a ball.

In light of Corollary (5.5), it is clear that the inequalities of Corollaries (6.4) and (6.3) make stronger statements than (imply) the classical inequalities (1.9) between the volume of a convex body and its projection measures. Specifically, if we combine the inequalities of Corollaries (6.4) and (5.5), we obtain

$$
\omega_{n}^{i+1} V(K)^{n-i-1} \leq \omega_{n}^{n+1} \omega_{n-1}^{-n} V\left(\Pi_{i}^{*} K\right)^{-1} \leq W_{i+1}^{n}(K) .
$$

The inequalities between the quantities on the extreme left and right are, of course, the classical inequalities (1.9).

If we combine Corollary (6.4) with (3.4) and use the Hölder inequality, we obtain the following inequality between the volume of $K$ and the power means of the girth functions of $K$ : then

(6.5) CoRollaRY. If $K$ is a convex body in $\mathbf{R}^{n}, 0<i<n-1$, and $p \geq-n$,

$$
\omega_{n-1}\left(\frac{V(K)}{\omega_{n}}\right)^{(n-i-1) / n} \leq\left[\frac{1}{n \omega_{n}} \int_{S^{n-1}} w_{i}\left(K^{u}\right)^{p} d S(u)\right]^{1 / p}
$$

with equality if and only if $K$ is a ball.

The case $i=0$ of Corollary (6.5) can be found in [24]. We note that the case $p=1$ in Corollary (6.5) gives the classical inequalities (1.9) between the volume of a convex body and its projection measures (viz. (2.9)). Because of Jensen's inequality, all of the cases with $-n \leq p<1$ are strengthened versions of the classical inequalities between the volume of a convex body and its projection measures.

The original motivation for much of the work presented in this article came from a desire on the part of the author to obtain the result given in Corollary (6.5).

The polar projection inequality was obtained by using the general centroid inequality. However, we note that if we were to start with the polar projection 
inequality and combine it with the polar centroid inequality, we would obtain, in turn, the general centroid inequality, along with (by using Lemma (6.2)) the equality conditions.

7. Mixed projection integrals. For convex bodies $K_{1}, \ldots, K_{n}$ in $\mathbf{R}^{n}$ and a fixed integer $r$ with $0 \leq r<n$, we define the $r$ th mixed projection integral of $K_{1}, \ldots, K_{n}$ by

$$
I_{r}\left(K_{1}, \ldots, K_{n}\right)=\frac{\omega_{n}^{n-r-2}}{n \omega_{n-1}^{n}} \int_{S^{n-1}} w_{r}\left(K_{1}^{u}\right) \cdots w_{r}\left(K_{n}^{u}\right) d S(u) .
$$

For $K_{1}=\cdots=K_{n}=B$, a trivial computation shows that

$$
I_{r}(B, \ldots, B)=\omega_{n}^{n-r-1} .
$$

We require a form of the Hölder inequality $[\mathbf{1 7}$, p. 140] that states that for positive continuous functions $f_{1}, \ldots, f_{n}$ on $S^{n-1}$,

$$
\int_{S^{n-1}} \prod_{i=1}^{n} f_{i}(u) d S(u) \leq \prod_{i=1}^{n}\left[\int_{S^{n-1}} f_{i}^{n}(u) d S(u)\right]^{1 / n}
$$

with equality if and only if all pairs $f_{i}, f_{j}$ are proportional. For $0 \leq r<n-1$, we take $f_{i}(u)=w_{r}\left(K_{i}^{u}\right)^{-1}$, apply the Hölder inequality, use (3.4) and obtain

$$
\left[\frac{1}{n} \int_{S^{n-1}}\left[w_{r}\left(K_{1}^{u}\right) \cdots w_{r}\left(K_{n}^{u}\right)\right]^{-1} d S(u)\right]^{n} \leq V\left(\Pi_{r}^{*} K_{1}\right) \cdots V\left(\Pi_{r}^{*} K_{n}\right),
$$

with equality if and only if (for all $i, j)$ the $(n-1-r)$-girth functions of $K_{i}$ and $K_{j}$ are proportional.

From Jensen's inequality, or from a simple application of the Schwarz inequality $[\mathbf{1 7}$, p. 132], we get

$$
n \omega_{n}^{n-r} \leq \omega_{n-1}^{n} I_{r}\left(K_{1}, \ldots, K_{n}\right) \int_{S^{n-1}}\left[w_{r}\left(K_{1}^{u}\right) \cdots w_{r}\left(K_{n}^{u}\right)\right]^{-1} d S(u) .
$$

If we combine (7.2), (7.3) and Corollary (6.4), we obtain

(7.4) THEOREM. If $K_{1}, \ldots, K_{n}$ are convex bodies in $\mathbf{R}^{n}$ and $0 \leq r<n-1$, then

$$
\left[V\left(K_{1}\right) \cdots V\left(K_{n}\right)\right]^{n-r-1} \leq I_{r}\left(K_{1}, \ldots, K_{n}\right)^{n},
$$

with equality if and only if the $K_{i}$ are balls.

The case $r=0$ of Theorem (7.4) was obtained in [24].

8. Circumscribing cylinders inequality. For a convex body $K$ and a direction $u \in S^{n-1}$, let $V_{K}(u)$ and $S_{K}(u)$ denote, respectively, the volume and lateral surface area of the right cylinder circumscribed about $K$ with bases orthogonal to $u$. Knothe $[\mathbf{1 8}]$ for $n=3$ and Chakerian $[\mathbf{1 1}]$ for $n \geq 3$ derived inequalities between the volume of $K$ and the arithmetic mean of $V_{K}(u)$, and between the surface area of $K$ and the arithmetic mean of $S_{K}(u)$. In $[\mathbf{2 4}]$ an inequality was obtained between $V(K)$ and the harmonic mean of $V_{K}(u)$. We now derive an inequality between $V(K)$ and the harmonic mean of $S_{K}(u)$.

By definition we have

$$
S_{K}(u)=(n-1) w_{1}\left(K^{u}\right) b(K, u),
$$


where $b(K, u)$ denotes the width of $K$ in the direction $u$ (distance between the supporting hyperplanes of $K$ that are orthogonal to $u$ ).

If $K$ is an arbitrary convex body in $\mathbf{R}^{n}(n>2)$, then as an immediate consequence of the Blaschke-Santaló inequality for centrally symmetric bodies (see [20]) we have

$$
2^{n} V(K) \leq \omega_{n}\left[\frac{1}{n \omega_{n}} \int_{S^{n-1}} b(K, u)^{-2} d S(u)\right]^{-n / 2},
$$

with equality if and only if (for $n>2$ ) $K$ is a ball.

If we use (8.1), apply the Schwarz inequality, and use (8.2) and Corollary (6.5), we obtain

(8.3) THEOREM. If $K$ is a convex body in $\mathbf{R}^{n}$, then

$$
2 \omega_{n-1}\left(\frac{V(K)}{\omega_{n}}\right)^{(n-1) / n} \leq\left[\frac{n-1}{n \omega_{n}} \int_{S^{n-1}} S_{K}(u)^{-1} d S(u)\right]^{-1}
$$

with equality if and only if $K$ is a ball.

\section{REFERENCES}

1. A. D. Aleksandrov, Zur Theorie der gemischten Volumina von konvexen Körpen. I. Verallgemeinerung einiger Begriffe der Theorie der konvexen Körper, Mat. Sb. (N.S.) 2 (1937), 947-972. (Russian with German summary)

2. __ Zur Theorie der gemischten Volumina von konvexen Körpern. II. Neue Ungleichungen zwrschen den gemischten Volumina und ihre Anwendungen, Mat. Sb. (N.S.) 2 (1937), 1205-1238. (Russian with German summary)

3. W. Blaschke, Vorlesungen über Differentialgeometrie. II, Springer-Verlag, Berlin, 1923.

4. E. D. Bolker, A class of convex bodies, Trans. Amer. Math. Soc. 145 (1969), 323-345.

5. T. Bonnesen and W. Fenchel, Theorie der konvexen Körper, Springer-Verlag, Berlin, 1934.

6. G. R. Burton, On the sum of a zonotope and an ellipsoid, Comment. Math. Helv. 51 (1976), 369-387.

7. Ju. D. Burago and V. A. Zalgaller, Geometric inequalities, "Nauka", Leningrad. Otdel., Leningrad, 1980. (Russian)

8. H. Busemann, Volume in terms of concurrent cross-sections, Pacific J. Math. 3 (1953), 1-12.

9. _ Convex surfaces, Interscience, New York, 1958.

10. G. D. Chakerian, Sets of comstant relative width and constant relative brightness, Trans. Amer. Math. Soc. 129 (1967), 26-37.

11. 12 (1972), 249-256.

12. _ Isoperimetric inequalities for the mean width of a convex body, Geom. Dedicata 1 (1973), 356-362.

13. __ Geometric inequalities for plane convex bodies, Canad. Math. Bull. 22 (1979), 9-16.

14. G. D. Chakerian and J. R. Sangwine-Yager, A generalization of Minkowski's inequality for plane comvex sets, Geom. Dedicata 8 (1979), 437-444.

15. W. Fenchel and B. Jessen, Mengenfunktionen und konvexe Körper, Danske Vid. Selskab. Mat.Fys. Medd. 16 (1938), 3.

16. H. Groemer, On multiple space subdivisions by zonotypes, Montash. Math. 86 (1978), 185-188.

17. G. H. Hardy, J. E. Littlewood and G. Pólya, Inequalities, Cambridge Univ. Press, Cambridge, 1934.

18. H. Knothe, Inversion of two theorems of Archimedes, Michigan Math. J. 4 (1957), 53-56.

19. K. Leichtweiss, Konvexe Mengen, Springer-Verlag, Berlin, 1980.

20. E. Lutwak, A general Bieberbach inequality, Math. Proc. Cambridge Philos. Soc. 78 (1975), 493-495.

21. __ Width-integrals of convex bodies, Proc. Amer. Math. Soc. 53 (1975), 435-439. 
22. _ _ Mixed width-integrals of convex bodies, Israel J. Math. 28 (1977), 249-253.

23. __ On the Blaschke-Santaló inequality, Discrete Geometry and Convexity (J. E. Goodman et al., eds.), N. Y. Acad. Sci. (in press).

24. __ A general isepiphanic inequality, Proc. Amer. Math. Soc. 90 (1984), 415-421.

25. P. McMullen, On zonotopes, Trans. Amer. Math. Soc. 159 (1971), 91-109.

26. C. M. Petty, Centroid surfaces, Pacific J. Math. 11 (1961), 1535-1547.

27. __ Projection bodies, Proc. Colloq. on Convexity (Copenhagen, 1965), 1967, pp. 234-241.

28. _ Isoperimetric problems, Proc. Conf. on Convexity and Combinatorial Geometry (Univ. of Oklahoma, June 1971), 1972, pp. 26-41 and 136.

29. __ Geominimal surface area, Geom. Dedicata 3 (1974), 77-97.

30. __ Affine isoperimetric problems, Discrete Geometry and Convexity (J. E. Goodman et al., eds.), N.Y. Acad. Sci. (in press).

31. C. A. Rogers, Sections and projections of convex bodies, Portugal. Math. 24 (1965), 99-103.

32. J. Saint Raymond, Sur le volume des corps convexes symétriques, Séminaire Choquet, 20e année (1980/81), Initiation à l'analyse, Comm. No. 11, 25 pp., Secrétariat Math., Paris, 1981.

33. L. A. Santaló, Un invariante afine para los cuerpos convexos del espacio de $n$ dimensiones, Portugal. Math. 8 (1949), 155-161.

34. R. Schneider, Zu einem Problem von Shephard über die Projectionen konvexer Körper, Math. Z. 101 (1967), 71-82.

35. __ Zonoids whose polars are zonoids, Proc. Amer. Math. Soc. 50 (1975), 365-368.

36. __ Rekonstruktion eines konvexen Körpers aus seinen Projektionen, Math. Nachr. 79 (1977), 325-329.

37. __ Curvature measures of convex bodies, Ann. Mat. Pura Appl. 116 (1978), 101-134.

38. ___ Random polytopes generated by anisotropic hyperplanes, Bull. London. Math. Soc. 14 (1982), 549-553.

39. ___ Random hyperplanes meeting a convex body, Z. Wahrsch. Verw. Gebiete 61 (1982), 379-387.

40. __ On the Aleksandrov-Fenchel inequality, Discrete Geometry and Convexity (J. E. Goodman et al., eds.), N.Y. Acad. Sci. (in press).

41., in preparation.

42. R. Schneider and W. Weil, Zonoids and related topics, Convexity and its Applications (P. M. Gruber and J. M. Wills, eds.), Birkhäuser Verlag, Basel, 1983, pp. 296-317.

43. G. C. Shephard, Convex bodies and convexity on Grassman cones. VIII. Projection functions of vector sums of convex sets, J. London Math. Soc. 39 (1964), 417-423.

44. W. Süss, Zusammensetzung von Eikörpern und homothetische Eiflächen, Tôhoku Math. J. 35 (1932), 47-50.

45. W. Weil, Kontinuierliche Linearkombination von Strecken, Math. Z. 148 (1976), 71-84.

46. __ Blaschkes Problem der Lokalen Charakterisierung von Zonoiden, Arch. Math. 29 (1977), 655-659.

47. __ Zonoide und verwandte Klassen konvexer Körper, Monatsh. Math. 94 (1982), 73-84.

48. H. S. Witsenhausen, Metric inequalities and the zonoid problem, Proc. Amer. Math. Soc. 40 (1973), 517-520.

49. _ A support characterization of the zonotopes, Mathematika 25 (1978), 13-16.

Department of Mathematics, Polytechnic Institute of New York, 333 Jay STREET, BROOKLYN, NEW YORK 11201 